\title{
Neutron Scattering on Impurity Nanoclusters in Gel Samples
}

\author{
V. B. Efimov, ${ }^{1,2}$ L. P. Mezhov-Deglin, ${ }^{2}$ C. D. Dewhurst, ${ }^{3}$ \\ A. V. Lokhov, ${ }^{2}$ and V. V. Nesvizhevsky ${ }^{3}$ \\ ${ }^{1}$ Physics Department, Lancaster University, Lancaster LA1 4YB, UK \\ ${ }^{2}$ Laboratory of Quantum Crystals, Institute of Solid State Physics RAS, Chernogolovka, Moscow District 142432, Russia \\ ${ }^{3}$ Institut Max von Laue-Paul Langevin, 71 Avenue des Martyrs, 38042 Grenoble, France \\ Correspondence should be addressed to V. B. Efimov; victor_efimov@yahoo.co.uk
}

Received 28 July 2014; Accepted 22 September 2014

Academic Editor: Guillaume Pignol

Copyright (C) 2015 V. B. Efimov et al. This is an open access article distributed under the Creative Commons Attribution License, which permits unrestricted use, distribution, and reproduction in any medium, provided the original work is properly cited. The publication of this article was funded by SCOAP ${ }^{3}$.

Results of recent SANS experiments with impurity-helium gel (IHG) samples in He-II are presented. We estimate the mean size of the impurity nanoparticles that form the frame of the IHG samples and discuss the possibility to use IHG samples for the production of ultracold neutrons (UCNs) in He-II cooled to the temperature of a few $\mathrm{mK}$, as well as the reflection of UCNs at any temperature. Our results indicate that the most promising materials for these purposes might be the heavy water gel samples with the mean sizes of $\mathrm{D}_{2} \mathrm{O}$ clusters of $d \sim 8 \mathrm{~nm}$ and the heavy alcohol gel samples with the mean sizes of clusters of $d \sim 15 \mathrm{~nm}$.

\section{Introduction}

Many current investigations with ultracold neutrons (UCNs) are limited by available UCN densities [1-3]. Intense efforts are undertaken all over the world to increase the UCN densities. The most promising techniques are based on downscattering of cold neutrons, mainly those with the wavelength of $8.9 \AA$, to the UCN energy due to phonon production in the bulk of superfluid He-II cooled below the temperature of $0.8 \mathrm{~K}$ or downscattering of neutrons with a broad initial energy spectrum in solid deuterium at the temperature of about $5 \mathrm{~K}[4]$.

Our primary goal in relation to the GRANIT project $[5,6]$ is to develop and model a new type of neutron moderator $[7,8]$ suitable for equilibrium cooling of UCNs in ultracold impurity-helium gel (IHG) matter. We will check the feasibility of the method based on equilibrium cooling of very cold neutrons (VCNs) in the process of their interaction with an IHG sample in superfluid He-II cooled below a temperature of a few $\mathrm{mK}$. An IHG sample could play the role of a moderator for (very) cold neutrons and convert them into UCNs due to their quasielastic scattering by impurity clusters which are weakly connected with each other inside the gel frame (the dispersion matter) as well as with the surrounding
He-II (the dispersion medium of the gel), and in this manner, the energy could be transferred from VCNs to the dispersion medium. It should be noted that in this consideration we suppose that the wavelength of incident VCNs is comparable to characteristic sizes of the impurity nanoclusters.

The main goal of the SANS experiments discussed below was to study the structure of the IHG samples and to estimate the mean sizes of the impurity nanoclusters which form a firm skeleton (frame) of the sample in superfluid He-II. The IHG samples were prepared of the impurity nanoparticles weakly interacting with each other and weakly absorbing neutrons, like those prepared from molecular gases $\mathrm{D}_{2}$ and $\mathrm{O}_{2}$ or liquids like $\mathrm{D}_{2} \mathrm{O}$ and $\mathrm{C}_{2} \mathrm{D}_{5} \mathrm{OD}$ [9-17].

Our preceding measurements of neutron transmission through a heavy water gel sample with the diameter of $2.5 \mathrm{~cm}$ in He-II at the temperature of $T=1.5 \mathrm{~K}$ have shown [18] that, with lowering the cold neutron velocity $v$ from $160 \mathrm{~m} / \mathrm{s}$ to $30 \mathrm{~m} / \mathrm{s}$, the angular distribution of the intensity of transmitted neutrons $I(\alpha)$ changed significantly: from near straightforward scattering at the velocities significantly larger than $60 \mathrm{~m} / \mathrm{s}$ to preferred backscattering for velocities significantly smaller than $60 \mathrm{~m} / \mathrm{s}$ (the corresponding transition neutron kinetic energy $\leq 0.2 \mathrm{~K}$ and the wavelength $\lambda \geq$ $6 \mathrm{~nm}$ ). In the case of transmission of a VCN beam through 
a massive IHG sample, with increasing the sample diameter $D$ from $2.5 \mathrm{~cm}$ to $25 \mathrm{~cm}$, one might hope that the mechanism of transmission of VCNs with a velocity slightly above this transition velocity through the sample would change from straight forward to diffusion propagation (note that $25 \mathrm{~cm}$ is the inner diameter of the vessel in an industrial $10 l$ cryostat for storage of liquid helium). The total content of the impurity molecules could reach $\sim 10^{20} \mathrm{~cm}^{-3}$, and supposing that about $\sim 1 \%$ of the impurity molecules is contained in particles of small sizes (up to 100 molecules in a single cluster), one can estimate that the number density of small clusters in the IHG sample could reach $N \sim 10^{16} \mathrm{~cm}^{-3}$. In the case of diffusion propagation of an VCN through a massive spherical sample with the diameter of $D=25 \mathrm{~cm}$, the effective length between two crossings of the neutron trajectory with walls of the vessel $L_{\text {eff }} \approx 1 / 3\left(D^{2} / L_{N}\right) \gg D$, while the distance between two elastic collisions of the neutron with nanoclusters in the bulk of the massive IHG sample $L_{N} \approx(1 / \sigma N) \ll D$. Though the probability of inelastic scattering of VCNs on single nanoclusters with the diameter $d \leq 1 \mathrm{~nm}$ is much smaller than the probability of elastic scattering and also could not be estimated reliably at this stage, the total number of collisions of the neutron with the impurity nanocluster particles in the volume of sample, proportional to the ratio $D / L_{n}$, could be large enough under certain conditions for effective cooling of neutrons to the bath temperature.

Thus, the primary focus of our participation in this GRANIT project includes a series of experiments relevant to the development of VCN moderators suitable for a new UCN source. We carried out a set of experiments to gain a better understanding of the properties and the behavior of IHG samples in He-II as a cold neutron moderator and UCN converter. The neutron studies are supported by $\mathrm{X}$-ray investigations [19] of the structure and the phase transitions in the highly dispersed icy powder which are created following the decay of the water and ethanol gel samples on heating them above the temperature of $5 \mathrm{~K}$ in $\mathrm{He}$ gas atmosphere.

\section{Experimental Technique and Results}

The investigation was carried out using the SANS spectrometer $\mathrm{D} 22$. The range of neutron wavelengths $\lambda$ was varied from $0.4 \mathrm{~nm}$ to $2.4 \mathrm{~nm}$. The main measurements were performed at the wavelength of $\lambda \approx 0.6 \mathrm{~nm}$. The spectrometer covers a total momentum transfer range $q$ from 0.003 to $0.8 \AA^{-1}$ (see Figure 1).

For these measurements, we designed and tested a special optical cryostat [20]. Samples with the volume of a few cubic $\mathrm{cm}(\sim 2.5 \mathrm{~cm}$ in diameter) were prepared in situ in a quartz glass cell filled with superfluid He-II at the temperature of $T \sim 1.6 \mathrm{~K}$. The process of sample preparation was controlled with a video camera installed outside the cryostat near the upper pairs of optical windows placed in the middle part of the cryostat. After preparation of a sample, the experimental cell was shifted down to the tail of the cryostat. All the pieces of the tail were produced from aluminum in order to decrease neutron scattering in the cryostat walls. The time of

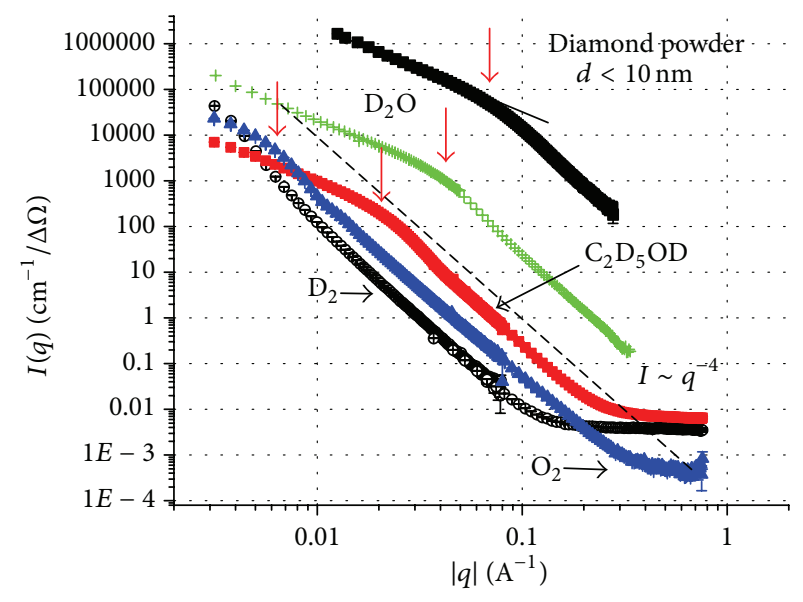

FIGURE 1: The intensity of small angle neutron scattering on impurities-helium gel samples and diamond nanopowder.

preparation of a sample was about 1 hour. The time of storage of superfluid He-II in the cryostat was longer than 10 hours.

The samples were prepared by means of condensation of a mixture of pure ${ }^{4} \mathrm{He}$ and the impurity vapors on the surface of He-II in the experimental quartz cell. For comparison, we have measured the scattering of neutrons on diamond nanopowder (by certificate, the size of diamond nanoparticles was $d \leq 10 \mathrm{~nm}$ ). The results of SANS measurements are shown in Figure 1. All these measurements were carried out in $\mathrm{He}$-II at a temperature of $T \sim 1.66 \mathrm{~K}$ except diamond powder. A thin layer of the commercial powder of nanodiamond [21] in an $\mathrm{Al}$ envelope was studied at the room temperature.

The dashed line in Figure 1 corresponds to the Porod law: $I(q) \sim q^{-4}$ provided the condition $q * d \gg 1$ [22]. The arrows above the curves $I(q)$ indicate the points of cusps, which were defined by approximating the slope of the $I(q)$ curves at small and large $q$ values.

Some results of numerical estimations of the intensity dependence $I(q)$ in the Born approximation [23] are shown in Figure 2. The dashed line corresponds to the Porod law $I \sim q^{-4}$; curve 1 shows scattering of neutrons on spherical nanoparticles with the radius of $R=10 \mathrm{~nm}$; curve 2 indicates results for the particle sizes distributed randomly from $1 \mathrm{~nm}$ to $19 \mathrm{~nm}$ (with the mean value $10 \mathrm{~nm}$ ); and curve 3 is for the Gaussian distribution with the mean size of $10 \mathrm{~nm}$. The change of the slope of the $I(q)$ curve at low $q$ values is attributed to the transition to the Guinier region [24] in the range of $q R_{g}<1$. Here, $R_{g}=(3 / 5)^{1 / 2} * R$ for spherical particles, and the particle diameter is $d<2.6 / q$. If the size of spherical particles $d>2.6 / q$, one could observe a number of interference minima in the $I(q)$ curve (1 curve in Figure 2). Whether the sizes of clusters are distributed randomly in the range from $1 \mathrm{~nm}$ to $19 \mathrm{~nm}$ (the mean value $10 \mathrm{~nm}$, curve 2) or assuming the Gaussian distribution (with the mean size $10 \mathrm{~nm}$ and the width of the distribution curve equal to $4.5 \mathrm{~nm}$ ) as shown in curve 3, the interference minima had disappeared and $I(q)$ became smooth enough. It is clear that with increasing the size of particles the kinks in the curves $I(q)$ are shifting to the lower $q$ wave range (curves 1, 3, and 2). 


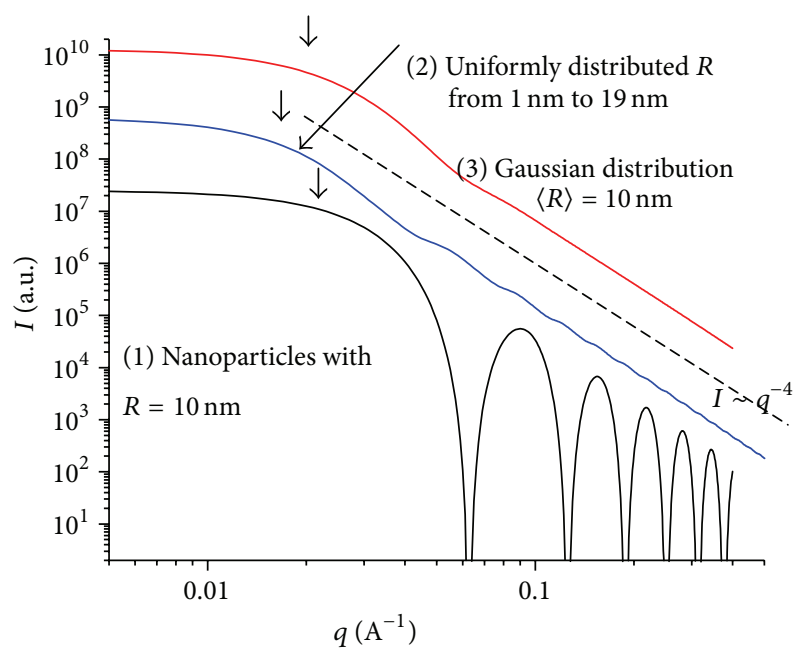

FIGURE 2: Results of calculation of neutron scattering in the Born approximation.

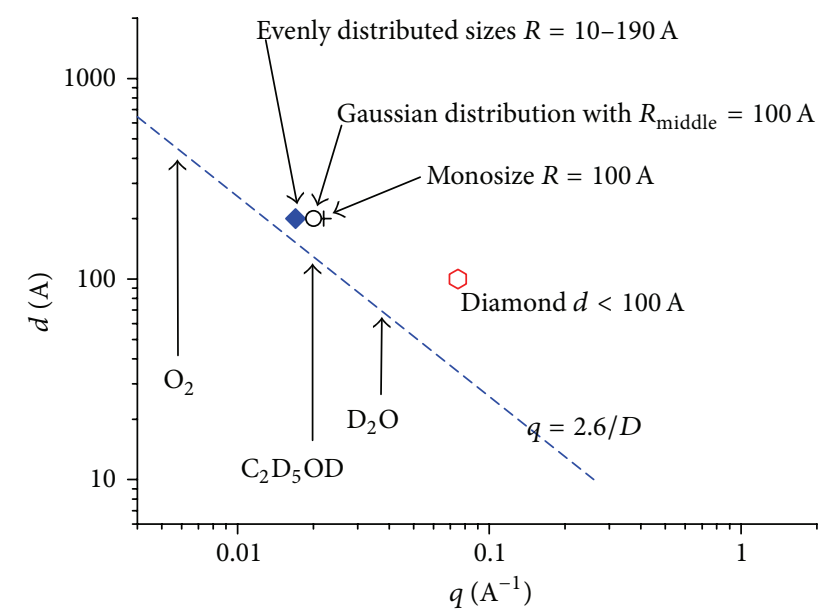

FIGURE 3: Estimation of sizes of nanoparticles in impurity-helium gels.

The results of our estimations of the size of nanoparticles in different IHG samples are plotted in Figure 3. The dotted line indicates the transition into the Guinier region. The points correspond to the positions of kinks in the calculation of the particle size distribution. The diamond point shows the position of the kink in the $I(q)$ curve for the diamond nanopowder (by the company certificate the size of particles was $d \leq 10 \mathrm{~nm})$.

As follows from Figure 3, the mean sizes of clusters in $\mathrm{D}_{2} \mathrm{O}$ gel samples were equal to $d \sim 8 \mathrm{~nm}$ and $d \sim$ $15 \mathrm{~nm}$ for the deuterated ethanol sample. It is interesting to note that mean sizes of the bcc crystals in the icy powder, created following the decay of the IHG samples, were close to these sizes, as it was observed in our X-rays studies [25]. The icy powder was warming up first to the liquid nitrogen temperature and then we studied the transitions from amorphous to nanocrystalline structures with further increasing the temperature of the sample. In the beginning, the total content of the amorphous phase in the water ice samples reached up to $40 \%$ in the normal water and up to $90 \%$ in the powder of heavy water. On heating the icy sample to the temperature of up to $110 \mathrm{~K}$ (for heavy water up to $130 \mathrm{~K}$ ), the amorphous phase was transformed to crystalline cubic phase and then we observed the transformation into the convenient hexagonal phase. As mentioned above, the sizes of initial cubic ice crystals were $6-10 \mathrm{~nm}$ [19]. In the case of ethanol samples, the transition from amorphous to monoclinic phase occurred at a temperature of $T \sim 110 \mathrm{~K}$ and the sizes of crystallites were close to $30-40 \mathrm{~nm}$.

\section{IHG Samples as Neutron Moderators}

As pointed out in [7], small sizes of impurity clusters weakly interacting with each other in the frame of the gel sample and with superfluid He-II in nanopores might be useful for developing VCN moderators of a new type, in which the energy of VCNs is transferred into the helium bath cooled down to the temperature of a few $\mathrm{mK}$ via their inelastic interaction with the clusters.

According to [8], the most interesting for these moderators could be $\mathrm{D}_{2} \mathrm{O}$ or the heavy alcohol $\mathrm{C}_{2} \mathrm{D}_{5} \mathrm{OD}$ gel samples. In principle, the total content of small (about $1 \mathrm{~nm}$ ) particles in bulk of these samples can be increased significantly on heating the freshly prepared sample in liquid helium up to $4 \mathrm{~K}$ and subsequent cooling [25].

The time-of-flight measurements of inelastic neutron scattering on these samples could give any answer on efficiency of the inelastic scattering of CNs and VCNs on the gel samples as well as on the gel samples prepared from other materials.

Elastic Neutron Scattering. The first experiment on VCN scattering yielded very encouraging results. It was observed that the beam transmission through a heavy water-helium gel sample with the volume of $\sim 3 \mathrm{~cm}^{3}$ in $\mathrm{He}$-II at the temperature $T \sim 1.6 \mathrm{~K}$ changed from $\sim 50 \%$ to $\sim 0 \%$ on decreasing the neutron velocity from $160 \mathrm{~m} / \mathrm{s}$ to $30 \mathrm{~m} / \mathrm{s}$. The angular distribution of neutron scattering changes as well: at higher neutron energies, the scattering is directed into the front hemisphere mainly $\left(<30^{\circ}\right)$, and at low energies, the angular distribution of scattered neutrons is close to spherical [18]. The transition from straight to diffusion propagation of VCNs in the bulk of a massive gel sample with increasing the sample volume with diameter up to $25 \mathrm{~cm}$ might result in a significant increase in the time of propagation of neutrons between two consequent crossings of the vessel walls and thus in a corresponding increase of the probability of decreasing the energy of neutrons down to the temperature of the He-II bath cooled below $10 \mathrm{mK}$.

\section{Conclusion}

We have made neutron scattering measurements on impurity-helium gels-a new class of "soft-matter." As an impurity, we used gases $\mathrm{D}_{2}$ and $\mathrm{O}_{2}$ and vapor of liquids $\mathrm{D}_{2} \mathrm{O}$ and $\mathrm{C}_{2} \mathrm{D}_{5} \mathrm{OD}$. The mean sizes of nanoparticles in the gels were $6-10 \mathrm{~nm}$ for heavy water gel and $15-30 \mathrm{~nm}$ for 
deuterated ethanol samples. We propose that the massive IHG samples could be promising for the purposes of VCN cooling down to UCNs.

\section{Conflict of Interests}

The authors declare that there is no conflict of interests regarding the publication of this paper.

\section{Acknowledgments}

The authors are grateful to Dr. R. May and Dr. A. Wiedenmann for help in the experiments. The work was supported in part by Grant RFBR-13-02-00378.

\section{References}

[1] J. Butterworth, P. Geltenbort, and E. Korobkina, "Proceedings of the International Workshop on Particle Physics with Slow Neutrons, Institut Laue-Langevin, Grenoble, France, October 22-24, 1998," Nuclear Instruments and Methods in Physics Research A, vol. 440, no. 3, p. 5, 2000.

[2] V. V. Nesvizhevsky, C. Plonka-Spehr, and K. Protasov, "Particle physics with slow neutrons. Proceedings of the International Workshop on Particle Physics with Slow Neutrons, Institut Laue-Langevin, Grenoble, France, May 29-31, 2008," Nuclear Instruments and Methods A, vol. 611, no. 2-3, p. 7, 2009.

[3] I. Antoniadis, S. Baessler, O. Bertolami et al., "Workshop GRANIT-2010, 14-19 February 2010, Les Houches, France," Comptes Rendus Physique, vol. 12, no. 8, pp. 703-706, 2011.

[4] R. Golub and J. M. Pendlebury, "Super-thermal sources of ultracold neutrons," Physics Letters A, vol. 53, no. 2, pp. 133-135, 1975.

[5] S. Baessler, M. Beau, M. Kreuz et al., "The GRANIT spectrometer," Comptes Rendus Physique, vol. 12, no. 8, pp. 707-728, 2011.

[6] D. Roulier, F. Vezzu, S. Baessler et al., "Status of the GRANIT facility," http://arxiv.org/abs/1410.1376.

[7] V. V. Nesvizhevsky, "Interaction of neutrons with nanoparticles," Physics of Atomic Nuclei, vol. 65, no. 3, pp. 400-408, 2002.

[8] V. V. Nesvizhevsky, G. Pignol, and K. V. Protasov, "Nanoparticles as a possible moderator for an ultracold neutron source," International Journal of Nanoscience, vol. 6, no. 6, pp. 485-499, 2007.

[9] V. Khmelenko, D. Lee, and S. Vasiliev, "Matrix isolation of $\mathrm{H}$ and D atoms: physics and chemistry from 1.5 to $0.1 \mathrm{~K}$," in Physics and Chemistry at Low Temperatures, L. Khriachtchev, Ed., chapter 5, Pan Stanford Publishing, Singapore, 2011.

[10] V. V. Khmelenko, E. P. Bernard, S. A. Vasiliev, and D. M. Lee, "Tunnelling chemical reactions of hydrogen isotopes in quantum solids," Russian Chemical Reviews, vol. 76, no. 12, pp. 1107-1121, 2007.

[11] L. P. Mezhov-Deglin, "Impurity nanocluster structures in liquid helium," Uspekhi Fizicheskikh Nauk, vol. 48, no. 10, pp. 10611070, 2005.

[12] V. Efimov and L. Mezhov-Deglin, "Method of preparation of nanoparticles from gases and vapor of fluids," Patent no. 2399581, 2010.

[13] E. B. Gordon and A. F. Shestakov, "Helium localization around the microscopic impurities embedded into liquid helium," Fizika Nizkikh Temperatur, vol. 26, no. 1, pp. 5-33, 2000.
[14] M. Barranco, R. Guardiola, S. Hernández, R. Mayol, J. Navarro, and M. Pi, "Helium nanodroplets: an overview," Journal of Low Temperature Physics, vol. 142, no. 1-2, pp. 1-81, 2006.

[15] S. I. Kiselev, V. V. Khmelenko, and D. M. Lee, "Sound propagation in liquid he in impurity-helium solids," Fizika Nizkikh Temperatur, vol. 26, no. 9-10, pp. 874-883, 2000.

[16] S. Kiselev, V. V. Khmelenko, D. M. Lee et al., "Structural studies of impurity-helium solids," Physical Review B, vol. 65, no. 2, Article ID 024517, 12 pages, 2001.

[17] S. Kiselev, V. V. Khmelenko, D. M. Lee et al., "X-ray studies of structural changes of impurity-helium solids," Journal of Low Temperature Physics, vol. 126, no. 1-2, pp. 235-240, 2002.

[18] L. P. Mezhov-Deglin, V. B. Efimov, A. V. Lokhov et al., "Scattering of cold neutrons on gel samples formed by impurity clusters in superfluid He-II," Journal of Low Temperature Physics, vol. 148, no. 5-6, pp. 833-837, 2007.

[19] V. B. Efimov, A. N. Izotov, A. A. Levchenko, L. P. MezhovDeglin, and S. S. Khasanov, "Structural transitions in ice samples at low temperatures and pressures," JETP Letters, vol. 94, no. 8, pp. 621-625, 2011.

[20] V. Efimov, L. Mezhov-Deglin, and A. Lokhov, "Combining cryostat for neutron investigation," PTE. In press.

[21] Diamond nanopowder 95 +\%, http://www.sigmaaldrich.com/.

[22] G. Porod, "Die Röntgenkleinwinkelstreuung von dichtgepackten kolloiden Systemen-I. Teil," Kolloid-Zeitschrift, vol. 124, no. 2, pp. 83-114, 1951.

[23] M. Born, "Zur Quantenmechanik der Stoßvorgänge," Zeitschrift für Physik, vol. 37, no. 12, pp. 863-867, 1926.

[24] A. Gunier and G. Fournet, Small Angle Scattering of X-Rays, John Wiley \& Sons, 1955.

[25] L. P. Mezhov-Deglin, V. B. Efimov, A. V. Lokhov et al., "Neutron studies of impurity gels of heavy water and deuterium in superfluid He-II," Journal of Low Temperature Physics, vol. 150, no. 3-4, pp. 206-211, 2008. 

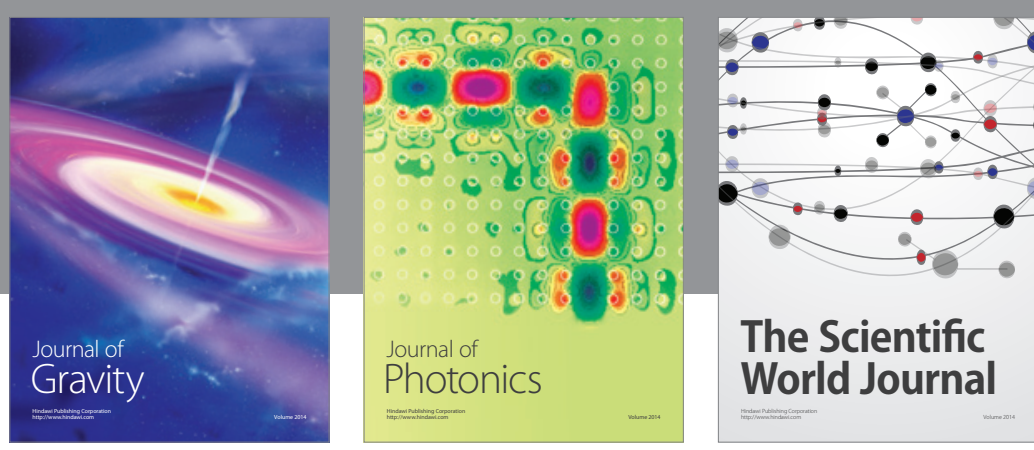

The Scientific World Journal
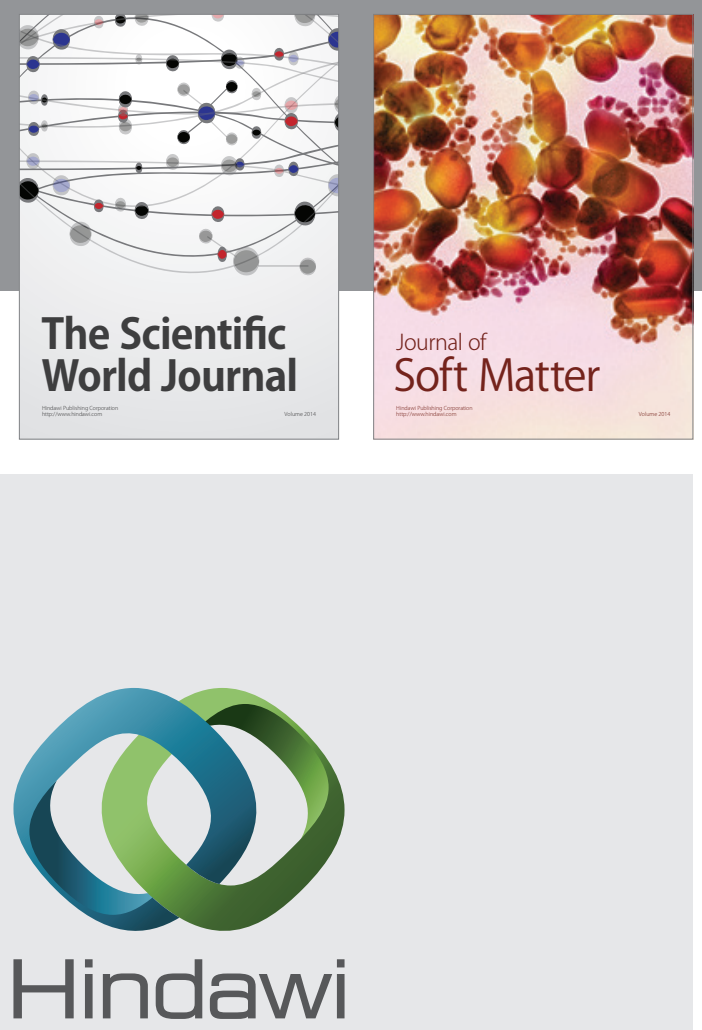

Submit your manuscripts at

http://www.hindawi.com

nternational Journal of

Statistical Mechanics
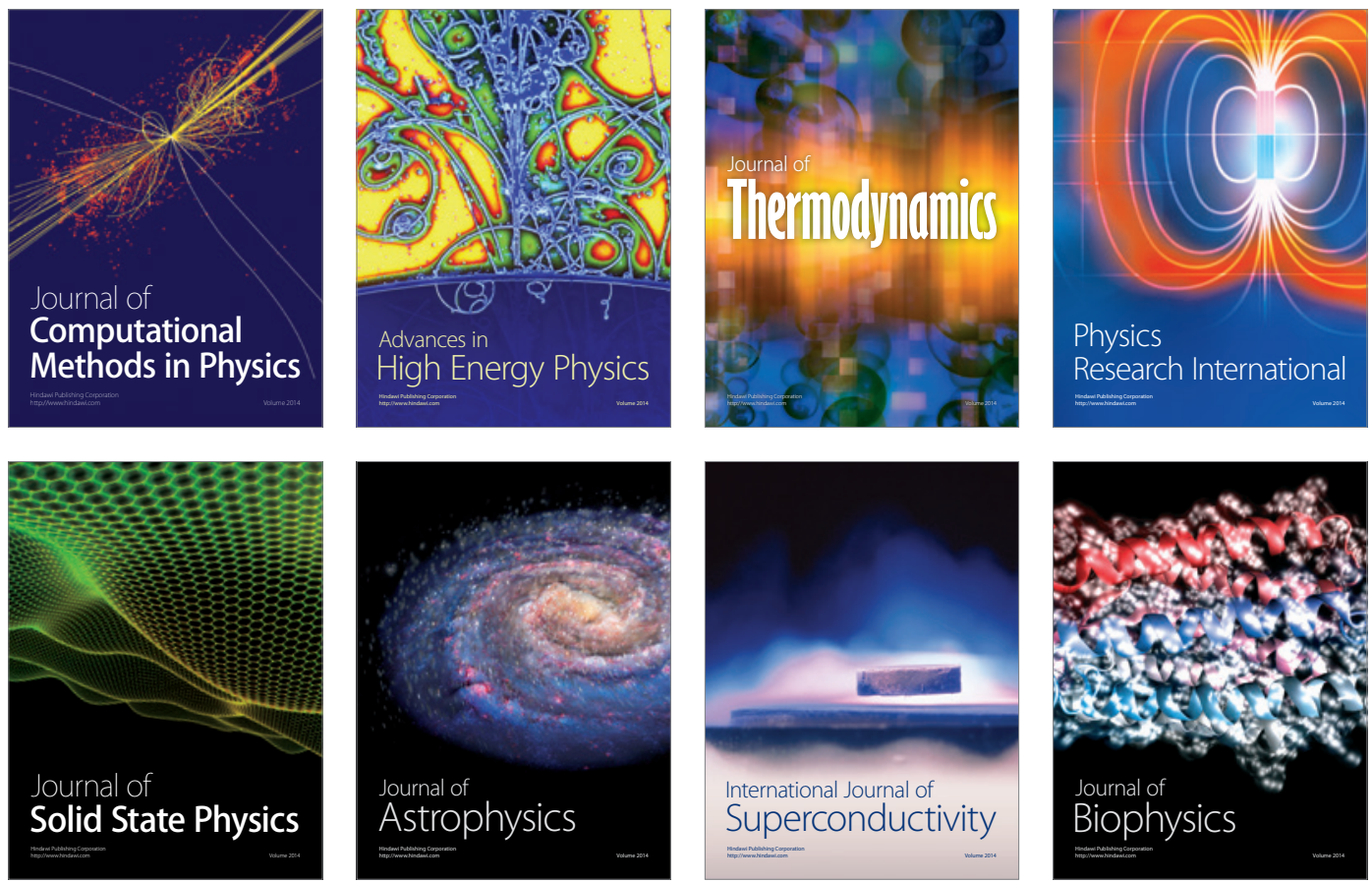
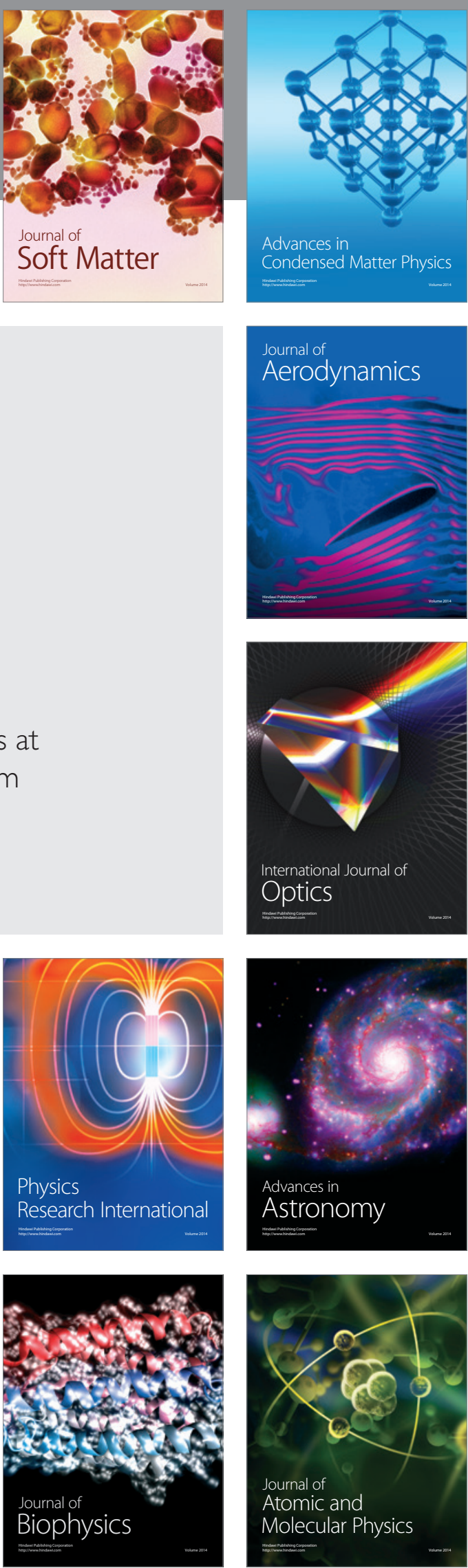